\title{
Pentoxifylline May Restore Kanamycin-Induced Renal Damage in Rats
}

\author{
Orhan Corum', Ozgur Ozdemir ${ }^{2}$, Mustafa Hitit ${ }^{3}$, Duygu Durna Corum', Devran Coskun ${ }^{4,5}$ \& Ayse Er ${ }^{4}$
}

\begin{abstract}
Background: Kidney damage can be caused by many factors, such as using certain drugs in high doses or over the long term. The use of one such group of drugs, aminoglycosides, which act as Gram-negative antibacterial therapeutic agents, can lead to nephrotoxicity. It has been hypothesized that aminoglycoside-induced nephrotoxicity might be prevented by using pentoxifylline, which has antioxidant and anti-inflammatory effects and improves microcirculation. The objective of this present research was to determine the protective effects of pentoxifylline on kanamycin-induced kidney damage.

Materials, Methods \& Results: Thirty-two male Wistar rats were divided into four groups as follows: control, pentoxifylline, kanamycin, and kanamycin + pentoxifylline. The control group received intraperitoneal (IP) injections of $0.5 \mathrm{~mL}$ normal saline solution once a day (d) (SID) for $20 \mathrm{~d}$; the pentoxifylline group received IP injections of $50 \mathrm{mg} / \mathrm{kg}$ pentoxifylline twice a day (BID) for $20 \mathrm{~d}$, the kanamycin group received subcutaneous (SC) injections of $500 \mathrm{mg} / \mathrm{kg}$ kanamycin SID for $20 \mathrm{~d}$, and the kanamycin + pentoxifylline group received both SC injections of $500 \mathrm{mg} / \mathrm{kg}$ kanamycin SID and IP injections of $50 \mathrm{mg} / \mathrm{kg}$ pentoxifylline BID for $20 \mathrm{~d}$. At the end of $20 \mathrm{~d}$, blood samples were taken from the heart by cardiac puncture under general anesthesia. After euthanizing the rats by cervical dislocation under anesthesia, the kidneys were immediately removed, relative kidney weights were calculated, and routine pathologic evaluations were conducted. Hemogram parameters were measured using a blood cell count apparatus and serum biochemical parameters were measured using an autoanalyzer. Kanamycin also caused $(P<0.05)$ tubular degeneration and tubular dilatation. Although pentoxifylline significantly reduced the level of kanamycin-induced tubular degeneration $(P<0.05)$, it did not significantly reduce tubular dilatation. Increases in relative kidney weights $(P<0.05)$ and in interstitial mononuclear cell $(\mathrm{MNC})$ infiltrates were observed in the kanamycin and kanamycin + pentoxifylline groups compared to those in the control and pentoxifylline groups. Statistically significant changes were determined in the levels of some hemogram and biochemical parameters within reference ranges $(P<0.05)$.

Discussion: In this study, both tubular degeneration and dilatation were observed in the kanamycin group. Pentoxifylline inhibited $(P<0.05)$ kanamycin-induced tubular degeneration and appeared to also reduce tubular dilatation, although this reduction was not significant. Tubular necrosis, epithelial edema of proximal tubules, tubular fibrosis, and perivascular inflammation might also be observed in aminoglycoside-induced nephrotoxicity. In current research, pentoxifylline prevented tubular damage induced by kanamycin, but did not inhibit infiltration by MNCs. Pentoxifylline also ameliorated amikacin- or gentamycin-induced histopathologic changes, especially those associated with tubular structures. The protective effects of pentoxifylline on kanamycin-induced tubular nephrotoxicity in this research might be a result of its stimulating the production of prostaglandin, a vasodilator, and of its improving microcirculation. Although the anti-inflammatory effects of pentoxifylline have been reported, these did not inhibit kanamycin-induced infiltration by interstitial MNCs in the present study. These results could indicate that the anti-inflammatory effects of pentoxifylline are not obvious and/or are dose dependent. Statistically significantly changes were determined in the levels of some hemogram and biochemical parameters in reference ranges. However, these changes were within the reference ranges for rats. These results suggested that kanamycin-induced tubular degeneration and dilatation might be prevented by administering pentoxifylline.
\end{abstract}

Keywords: pentoxifylline, kidney damage, kanamycin, hemogram, biochemical. 


\section{INTRODUCTION}

Kanamycin is an aminoglycoside antibiotic. Aminoglycosides are widely used for treating severe infections caused by aerobic Gram-negative bacteria, such as Enterobacteriaceae and Pseudomonas spp. However, there are some serious side effects of these antibiotics, nephrotoxicity being the most common. Parenteral-administered aminoglycosides accumulate within the lysosomes, Golgi apparatus, and endoplasmic reticulum in the renal cortex; consequently, result in renal tubular dysfunction, the most common side effect, with the release of reactive oxygen species combined with cell apoptosis and necrosis $[8,9,21]$.

Pentoxifylline is a methylxanthine derivative and a nonspecific phosphodiesterase inhibitor. It is used mainly for treating peripheral vascular diseases by reducing blood viscosity; furthermore, pentoxifylline has many different pharmacologic uses as an anti-inflammatory, antioxidant, promoter of microcirculation, and inhibitor of platelet aggregation $[6,7,9,13,15]$. However, pentoxifylline's antioxidant effect is controversial and/or dose dependent $[2,26]$. In addition, it has been reported that positive effects of this drug might be observed after using it to treat diabetic nephropathy and acute kidney injury $[9,15]$. These positive effects might be the result of its stimulation of vasodilator prostaglandins. Some researchers have investigated the protective effects of pentoxifylline on aminoglycoside-induced nephrotoxicity [9].

Taking into consideration the antioxidant, vasodilator, and renoprotective properties of pentoxifylline $[2,6,7,9,15]$, this study aimed to determine the effects of pentoxifylline in the prevention of kanamycin-induced nephrotoxicity in rats.

\section{MATERIALS AND METHODS}

Animals

In the current research, a total of 32 male Wistar rats (12-14 weeks old, $234.63 \pm 2.68 \mathrm{~g}$ ) were used. The study protocol was approved by Ethics Committee of Experimental Medicine Research and Application Centre of Selcuk University (SUDAM). During the experimental period, the rats were housed in standard rat cages and allowed water and food ad libitum. The rats were caged in a central facility under controlled conditions ( $12 \mathrm{~h} \mathrm{light/dark} \mathrm{cycle} \mathrm{and} \mathrm{room} \mathrm{temperature}$ of $20^{\circ} \mathrm{C} \pm 2{ }^{\circ} \mathrm{C}$ ) at SUDAM.

\section{Experimental procedure}

The 32 rats were randomly divided into 4 groups as follows: control $(n=6)$, pentoxifylline $(n=6)$, kanamycin $(n=10)$, and kanamycin + pentoxifylline $(n=$ 10). The control group received an intraperitoneal (IP) injection of $0.5 \mathrm{~mL}$ normal saline solution once/d (SID) for $20 \mathrm{~d}$, the pentoxifylline group received an IP injection of $50 \mathrm{mg} / \mathrm{kg}$ pentoxifylline twice/d (BID) for $20 \mathrm{~d}$ $\left(\text { Trental amp }{ }^{\mathrm{TM}}\right)^{1}$ [3], the kanamycin group received a subcutaneous injection of $500 \mathrm{mg} / \mathrm{kg}$ kanamycin SID for $20 \mathrm{~d}$ (Kanovet enj sol $\left.{ }^{\mathrm{TM}}\right)^{2}$ [14], and the kanamycin + pentoxifylline group received an SC injection of $500 \mathrm{mg} /$ $\mathrm{kg}$ kanamycin SID for $20 \mathrm{~d}$ together with an IP injection of $50 \mathrm{mg} / \mathrm{kg}$ pentoxifylline BID for $20 \mathrm{~d}$. At the end of the $20 \mathrm{~d}$ period, blood samples were taken from the heart by cardiac puncture under general anesthesia $(60 \mathrm{mg} / \mathrm{kg}$ sodium thiopental, IP, Pental sodium) ${ }^{3}$. After the blood samples were taken, the rats were euthanized by cervical dislocation under general anesthesia. The kidney tissues were immediately collected after euthanasia, and stored at $-80^{\circ} \mathrm{C}$ until used.

\section{Biochemical and hematological measurements}

Serum biochemical parameters (urea, creatinine, AST, ALT, ALP, total protein, albumin, triglyceride, cholesterol, and total bilirubin) were measured on an autoanalyzer $^{4}$, while hemogram parameters (WBC, RBC, platelet, hemoglobin, hematocrit) were measured using a blood cell count apparatus (BC-2800 Auto Hematology Analyzer)5.

\section{Histopathological examination}

A systematic necropsy was conducted on the euthanized rats. The kidneys were immediately removed, weighed, and preserved in $10 \%$ formalin and the relative kidney weight (RKW) was calculated. The imbedded samples were cut into 5 - $\mu \mathrm{m}$ sections for routine histopathologic examination. The samples were stained with hematoxylin-eosin and evaluated using a light microscope. Lesions were scored as mild $(+1)$, moderate $(+2)$, and severe $(+3)$.

\section{Statistical analyses}

Research data are presented as the mean \pm standard error. Biochemical parameters, hemogram parameters, and RKW were analyzed using ANOVA and Duncan's post hoc test. The kidney damage scores were evaluated using the Mann-Whitney U test. SPSS $22.0^{6}$ was used for all statistical analyses. $P<0.05$ was considered statistically significant. 


\section{RESULTS}

The kidney damage scores and RKWs are shown in Table 1; serum biochemical and hemogram values are provided in Tables 2 and 3, respectively. Macroscopic and histopathologic observations are presented in Figures 1 and 2, respectively.

When the kidneys were macroscopically examined, enlarged, bulging, and pale kidneys were observed in the kanamycin group (Figure 1), while normal kidneys were observed in all other groups. The observed tubular degeneration in the kanamycin group was statistically different $(P<0.05)$ from that in the control and kanamycin + pentoxifylline groups, while the observed tubular dilatation in the kanamycin group was statistically different $(P<0.05)$ from that in the control and pentoxifylline groups (Table 1, Figure 2). Pentoxifylline reduced kanamycin-induced tubular dilatation, although the change was not sig- nificant. Interstitial MNC infiltrates were observed in the kanamycin and kanamycin + pentoxifylline groups (Figure 2), and kidney weights increased $(P<0.05)$ in these same groups relative to those in the control and pentoxifylline groups.

Creatinine, total protein, albumin, and cholesterol levels in the kanamycin + pentoxifylline group were statistically lower than other groups $(P<0.05$, Table 2), and an increase in total bilirubin levels were observed in the kanamycin group relative to that in the control and kanamycin + pentoxifylline groups $(P$ $<0.05$, Table 2$)$. In addition, triglyceride levels were lower $(P<0.05)$ in the kanamycin + pentoxifylline group than in the control group (Table 2), and WBC, $\mathrm{RBC}$, hemoglobin, and hematocrit levels were highest in the control group $(P<0.05)$. Statistically significant fluctuations were determined $(P<0.05)$ in platelet levels in all experimental groups (Table 3 ).

Table 1. Renal histopathologic scores and relative kidney weight in the experimental groups of male Wistar rats (mean $\pm \mathrm{SE}$ ).

\begin{tabular}{ccccc}
\hline Parameter & Control & Pentoxifylline & Kanamycin & Kanamycin + Pentoxifylline \\
\hline Relative kidney weight & $0.37 \pm 0.02^{\mathrm{b}}$ & $0.40 \pm 0.01^{\mathrm{b}}$ & $0.53 \pm 0.02^{\mathrm{a}}$ & $0.56 \pm 0.02^{\mathrm{a}}$ \\
Tubular degeneration & $0.67 \pm 0.11^{\mathrm{b}}$ & $0.80 \pm 0.12^{\mathrm{ab}}$ & $1.22 \pm 0.17^{\mathrm{a}}$ & $0.56 \pm 0.11^{\mathrm{b}}$ \\
Tubular dilatation & $0.00 \pm 0.00^{\mathrm{b}}$ & $0.10 \pm 0.10^{\mathrm{b}}$ & $0.83 \pm 0.14^{\mathrm{a}}$ & $0.38 \pm 0.16^{\mathrm{ab}}$ \\
Glomerular hypertrophy & $0.00 \pm 0.00$ & $0.30 \pm 0.12$ & $0.11 \pm 0.07$ & $0.25 \pm 0.09$ \\
Increase in glomerular mesangial cells & $0.00 \pm 0.00$ & $0.00 \pm 0.00$ & $0.28 \pm 0.09$ & $0.25 \pm 0.09$ \\
Interstitial MNC infiltration & $0.00 \pm 0.00^{\mathrm{b}}$ & $0.00 \pm 0.00^{\mathrm{b}}$ & $0.44 \pm 0.18^{\mathrm{ab}}$ & $0.69 \pm 0.09^{\mathrm{a}}$ \\
Enlargement in Bowman's space & $0.00 \pm 0.00$ & $0.00 \pm 0.00$ & $0.44 \pm 0.13$ & $0.31 \pm 0.16$ \\
\hline
\end{tabular}

${ }_{\mathrm{a}, \mathrm{b}}$ Different letters in the same line were statistically significant $(P<0.05)$. MNC: Mononuclear cell.

Table 2. Biochemical values in the experimental groups of male Wistar rats (mean $\pm \mathrm{SE})$.

\begin{tabular}{ccccc}
\hline Parameter & Control & Pentoxifylline & Kanamycin & Kanamycin + Pentoxifylline \\
\hline Urea $(\mathrm{mg} / \mathrm{dL})$ & $43.3 \pm 2.03$ & $45.8 \pm 2.95$ & $48.9 \pm 2.21$ & $47.0 \pm 1.03$ \\
Creatinine $(\mathrm{mg} / \mathrm{dL})$ & $0.59 \pm 0.02^{\mathrm{a}}$ & $0.60 \pm 0.03^{\mathrm{a}}$ & $0.63 \pm 0.03^{\mathrm{a}}$ & $0.48 \pm 0.04^{\mathrm{b}}$ \\
AST (U/L) & $188 \pm 23.1$ & $170 \pm 15.7$ & $242 \pm 41.7$ & $185 \pm 87.8$ \\
ALT (U/L) & $59.7 \pm 3.25$ & $55.3 \pm 2.49$ & $123 \pm 29.6$ & $89 \pm 47.8$ \\
ALP (U/L) & $215 \pm 17.6$ & $191 \pm 22.8$ & $219 \pm 26.4$ & $134 \pm 27.9$ \\
Total protein (g/dL) & $5.65 \pm 0.18^{\mathrm{a}}$ & $5.28 \pm 0.16^{\mathrm{a}}$ & $5.27 \pm 0.16^{\mathrm{a}}$ & $3.19 \pm 0.50^{\mathrm{b}}$ \\
Albumin (g/dL) & $3.08 \pm 0.05^{\mathrm{a}}$ & $2.88 \pm 0.10^{\mathrm{a}}$ & $2.92 \pm 0.09^{\mathrm{a}}$ & $1.81 \pm 0.26^{\mathrm{b}}$ \\
Triglyceride (mg/dL) & $118 \pm 7.30^{\mathrm{a}}$ & $109 \pm 12.3^{\mathrm{ab}}$ & $100 \pm 10.4^{\mathrm{ab}}$ & $83.8 \pm 6.80^{\mathrm{b}}$ \\
Cholesterol $(\mathrm{mg} / \mathrm{dL})$ & $56.5 \pm 2.40^{\mathrm{a}}$ & $50.8 \pm 5.52^{\mathrm{a}}$ & $59.8 \pm 3.70^{\mathrm{a}}$ & $36.7 \pm 5.12^{\mathrm{b}}$ \\
Total bilirubin $(\mathrm{mg} / \mathrm{dL})$ & $0.16 \pm 0.01^{\mathrm{c}}$ & $0.22 \pm 0.01^{\mathrm{ab}}$ & $0.25 \pm 0.01^{\mathrm{a}}$ & $0.19 \pm 0.02^{\mathrm{bc}}$ \\
\hline a,b,c Different letters in the same line were statistically significant $(P<0.05)$. AST: Aspartate aminotransferase, ALT: Alanine aminotransferase; ALP: \\
Alkaline phosphatase.
\end{tabular}


O. Corum, O. Ozdemir, M. Hitit, et al. 2018. Pentoxifylline May Restore Kanamycin Induced Renal Damage in Rats. Acta Scientiae Veterinariae. 46: 1564.

Table 3. Hemogram values in the experimental groups of male Wistar rats (mean $\pm \mathrm{SE}$ ).

\begin{tabular}{ccccc}
\hline Parameter & Control & Pentoxifylline & Kanamycin & Kanamycin + Pentoxifylline \\
\hline WBC $\left(\times 10^{9} / \mathrm{L}\right)$ & $13.4 \pm 0.34^{\mathrm{a}}$ & $10.4 \pm 0.87^{\mathrm{b}}$ & $10.5 \pm 0.52^{\mathrm{b}}$ & $8.47 \pm 0.57^{\mathrm{c}}$ \\
RBC $\left(\times 10^{12} / \mathrm{L}\right)$ & $9.33 \pm 0.11^{\mathrm{a}}$ & $8.25 \pm 0.20^{\mathrm{b}}$ & $7.48 \pm 0.15^{\mathrm{c}}$ & $7.68 \pm 0.16^{\mathrm{c}}$ \\
Platelet $\left(\times 10^{9} / \mathrm{L}\right)$ & $511 \pm 17.3^{\mathrm{b}}$ & $637 \pm 40.0^{\mathrm{a}}$ & $644 \pm 43.4^{\mathrm{a}}$ & $613 \pm 20.4^{\mathrm{ab}}$ \\
Hemoglobin $(\mathrm{g} / \mathrm{L})$ & $17.1 \pm 0.22^{\mathrm{a}}$ & $15.1 \pm 0.27^{\mathrm{b}}$ & $13.5 \pm 0.23^{\mathrm{c}}$ & $14.0 \pm 0.26^{\mathrm{c}}$ \\
Hematocrit $\%$ & $56.2 \pm 1.06^{\mathrm{a}}$ & $51.0 \pm 1.11^{\mathrm{b}}$ & $44.9 \pm 0.78^{\mathrm{c}}$ & $46.1 \pm 0.97^{\mathrm{c}}$ \\
\hline
\end{tabular}

a,b,c Different letters in the same line were statistically significant $(P<0.05)$. WBC: White blood cell; RBC: Red blood cell.

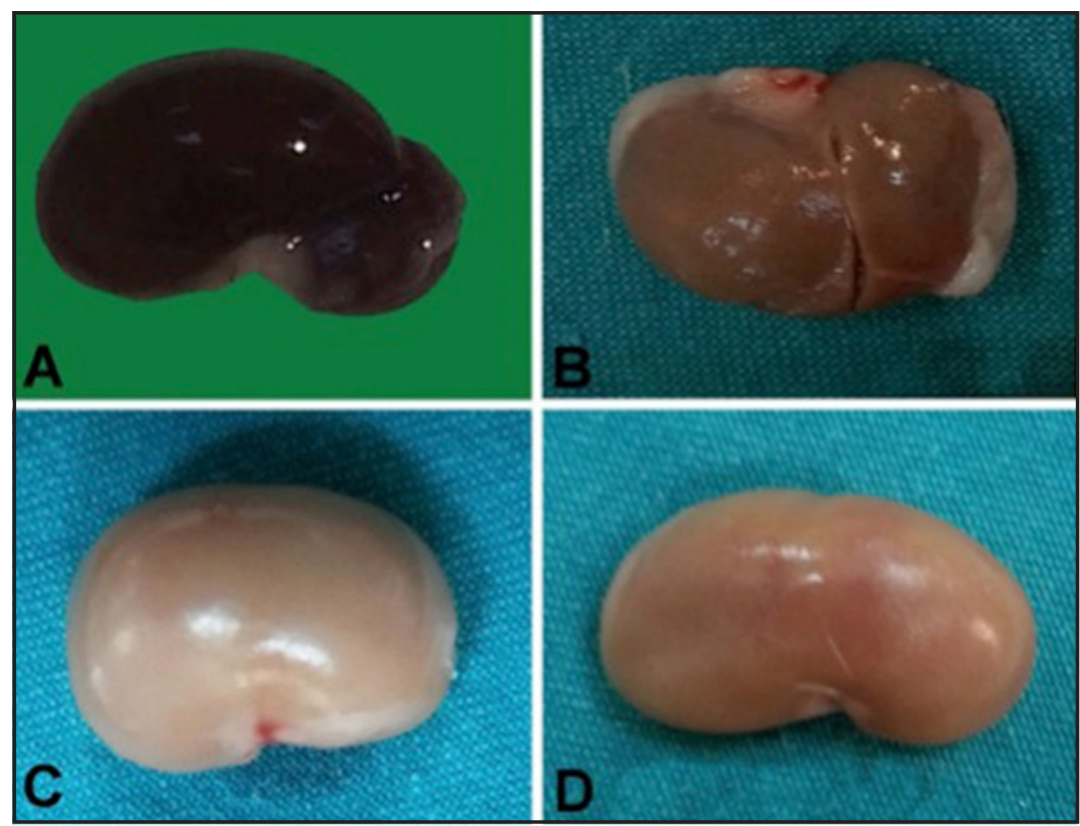

Figure 1. Macroscopic observations. A- Control group. B- pentoxifylline group. C- Kanamycin group (enlarged, pale, and swollen kidney). D- Kanamycin + pentoxifylline group (normal size and colored kidney).

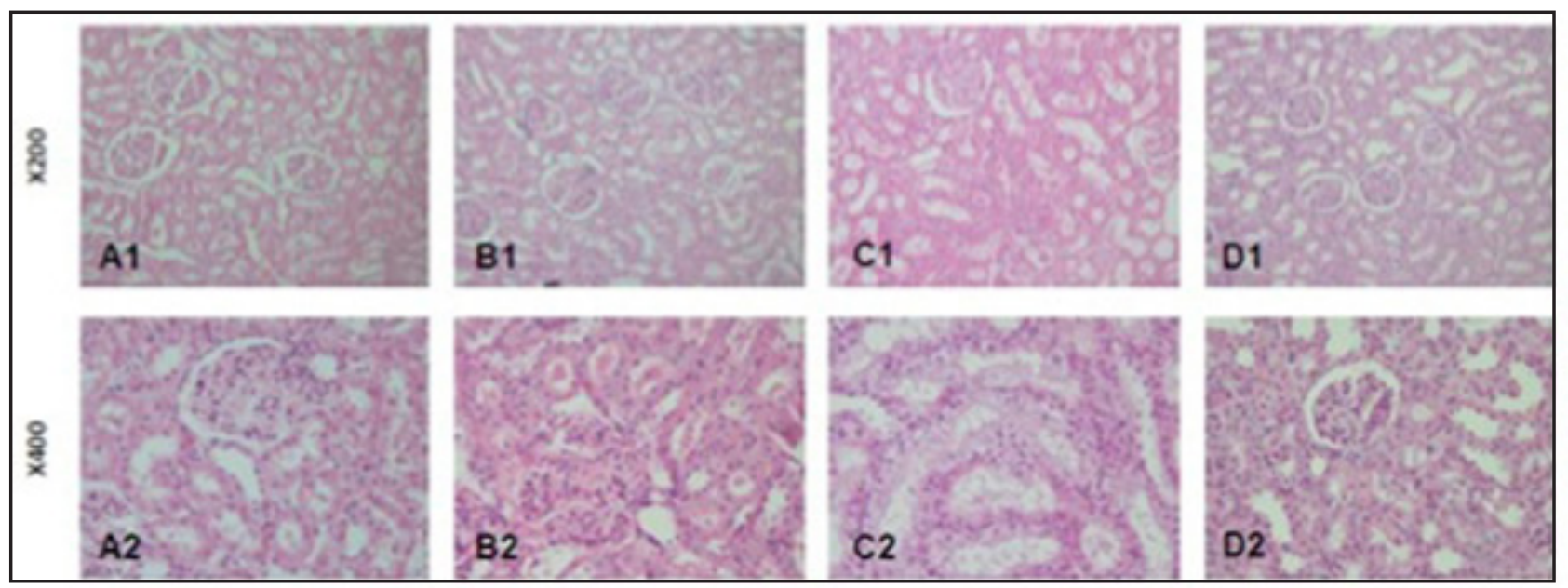

Figure 2. Kidney with hematoxylin-eosin staining. A\&B- Normal histopathological appearance. C- Tubular dilatation, tubular degeneration, and mononuclear cell infiltration. D- Decreased tubular score, but still the appearance of mononuclear cell infiltrates. 


\section{DISCUSSION}

The most common side effect of kanamycin is nephrotoxicity [2-9] and the drug accumulates in the kidneys 10-50 times more than in the plasma. Although it has been reported that kidney damage occurred in 25 $50 \%$ of patients, pentoxifylline might show a renoprotective effect in some diseases resulting from its vasodilator, antioxidant, and microcirculatory features [9,11,15,22].

In this study, no macroscopic and microscopic pathologies were observed in the control group (Table 1 , Figures $1 \& 2)$. The RKWs increased $(P<0.05)$ in the kanamycin and kanamycin + pentoxifylline groups (Table 1, Figure 1). Both tubular degeneration and dilatation were observed in the kanamycin group. Pentoxifylline inhibited $(P<0.05)$ kanamycin-induced tubular degeneration and appeared to also reduce tubular dilatation, although this reduction was not significant (Table 1, Figure 2). Parameters for glomerular hypertrophy, an increase in glomerular mesangial cells, and enlargement in Bowman's space did not differ significantly between the kanamycin and kanamycin + pentoxifylline groups. Tubular necrosis, epithelial edema of proximal tubules, tubular fibrosis, and perivascular inflammation might also be observed in aminoglycoside-induced nephrotoxicity [9]. Pentoxifylline produces no histopathological changes or an induction of apoptosis in the kidneys [3], while necrosis and apoptosis of tubular epithelial cells, tubular dilatation, increased connective tissue mass in some areas of the interstitium, an enlarged glomerulus or Bowman's capsule, neutrophil or MNC infiltrates, and hemorrhage in some areas of the interstitium have been reported in amikacin- or gentamycin-induced nephrotoxicity $[3,12,16,17]$. In the current research, pentoxifylline prevented tubular damage induced by kanamycin, but did not inhibit infiltration by MNCs (Table 1, Figure 2). The protective effects of pentoxifylline have been investigated in aminoglycoside-induced nephrotoxicity, and renoprotective effects of pentoxifylline have been reported [6,9]. Pentoxifylline also ameliorated amikacin- or gentamycin-induced histopathologic changes, especially those associated with tubular structures $[12,13,16,17]$. The protective effects of pentoxifylline on kanamycin-induced tubular nephrotoxicity in this research might be a result of its stimulating the production of prostaglandin, a vasodilator, and of its improving microcirculation $[9,15]$. Although the anti-inflammatory effects of pentoxifylline have been reported $[9,15]$, these did not inhibit kanamycin-induced infiltration by inter- stitial MNCs in the present study (Table 1, Figure 2). These results could indicate that the anti-inflammatory effects of pentoxifylline are not obvious and/or are dose dependent.

Statistically significantly changes $(P<0.05)$ were determined in the levels of creatinine, total protein, albumin, cholesterol, triglycerides, and total bilirubin in within-group comparisons (Table 2). Aminoglycoside antibiotics might cause statistically significant differences within reference ranges in serum biochemical values in rodents [25], while pentoxifylline did not show a dominant effect on these biochemical values in rodents [24]. In the current research, statistically determined changes in the damage markers of the kidney, liver, and bile duct [18] and in lipid metabolism values were within the reference ranges for rats $[1,4,10,18,20,23,27]$. These results might indicate that kanamycin and pentoxifylline have no undesirable effects on these functions in rats.

Statistically significant $(P<0.05)$ changes were determined in the WBC, RBC, platelet, hemoglobin, and hematocrit levels in the present research (Table $3)$; however, these changes were within the reference ranges for rats $[10,23]$. These results might indicate that kanamycin and pentoxifylline have no undesirable effects on hemogram values that reflect bone marrow function $[5,19]$ in rats.

\section{CONCLUSIONS}

Repetitive administration of kanamycin increased RKW and lead to histopathological tubular degeneration, tubular dilatation, and changes in interstitial MNC infiltrate levels. Although administration of kanamycin together with pentoxifylline prevented tubular damage and tubular dilatation, it had no effect on the other parameters. The effects of kanamycin together with pentoxifylline on hematological and biochemical parameters remained within the reference ranges. Additional molecular studies using different doses and time intervals are necessary to determine the potential effects of pentoxifylline on kanamycin-induced nephrotoxicity.

\section{MANUFACTURERS}

${ }^{1}$ Sanofi Aventis Drugs Ltd,. Istanbul, Turkey.

${ }^{2}$ Vetas Veterinary Drug. Istanbul, Turkey.

${ }^{3}$ IE Ulagay. Istanbul, Turkey.

${ }^{4}$ ILab-300 BioMérieux Diagnostics. Milan, Italy.

${ }^{5}$ Shenzen Mindray Bio-Medical Electronics Co., Ltd. Shenzen, China. ${ }^{6}$ IBM Corporation. Armonk, NY, USA. 
Acknowledgements. We thank Dr. Enver Yazar for scientific assistance. The study abstract was presented as a poster that the First International Turkish Veterinary Internal Medicine Congress on October 10-12, 2017, in Antalya, Turkey.

Ethical approval. The study protocol was approved by Ethics Committee of Experimental Medicine Research and Application Centre of Selcuk University (SUDAM, 2015/60).
Declaration of interest. The authors report no conflicts of interest. The authors alone are responsible for the content and writing of this paper.

\section{REFERENCES}

1 Er A., Dik B., Cetin G., Altan F., Uney K., Elmas M. \& Yazar E. 2013. Etanercept - Can It be Used in the Treatment of Endotoxemia? Kafkas Universitesi Veteriner Fakültesi Dergisi. 19: 133-139.

2 Horvath B., Marton Z., Halmosi R., Alexy T., Szapary L., Vekasi J., Biro Z., Habon T., Kesmarky G. \& Toth K. 2002. In vitro antioxidant properties of pentoxifylline, piracetam, and vinpocetine. Clinical Neuropharmacology. 25: $37-42$.

3 Kasap B., Turkmen M., Kiray M., Kuralay F., Soylu A., Tugyan K. \& Kavukcu S. 2013. Effects of pentoxifylline on gentamicin-induced nephrotoxicity. Renal Failure. 35: 1376-1381.

4 Kerr M.G. 2002. Clinical biochemistry. In: Veterinary Laboratory Medicine. 2nd edn. Ames: Blackwell Publishing Ltd., pp.69-198.

5 Kerr M.G. 2002. Haemotoloyg. In: Veterinary Laboratory Medicine. 2nd edn. Ames: Blackwell Publishing Ltd., pp.1-65.

6 Leporini C., Pisano A., Russo E., D’Arrigo G., DeSarro G., Copplino G. \& Bolignano. 2016. Effect of pentoxifylline on renal outcomes in chronic kidney disease patients: A systematic review and meta-analysis. Pharmacological Research. 107: 315-332.

7 Lyons A.J. \& Brennan P.A. 2017. Pentoxifylline - a review of its use in osteoradionecrosis. British Journal of Oral and Maxillofacial Surgery. 55: 230-234.

8 McWilliam S.J., Antoine D.J., Smyth R.L. \& Pirmohamed M. 2016. Aminoglycoside-induced nephrotoxicity in children. Pediatric Nephrology. 15: 1-11.

9 Nasiri-Toosi Z., Dashti-Khavidaki S., Khalili H. \& Lessan-Pezeshki M. 2013. A review of the potential protective effects of pentoxifylline against drug-induced nephrotoxicity. European Journal of Clinical Pharmacology. 69: 10571073 .

10 Ness R.D. 2004. Rodents. In: Carpenter JW. (Ed). Exotic Animal Formulary. London: Elsevier Saunders, pp.377-410.

11 Oliveira J.F.P., Cipullo J.P. \& Burdmann E.A. 2006. Aminoglycoside nephrotoxicity. Brazilian Journal of Cardiovascular Surgery. 21: 444-452.

12 Ozer M.K., Asci H., Oncu M., Yesilot S., Savran M., Bayram D. \& Cicek E. 2009. Effects of Pentoxifylline on Amikacin-Induced Nephrotoxicity in Rats. Renal Failure. 31: 134-139.

13 Pascarella L. \& Shortell C.K. 2015. Medical management of venous ulcers. Seminars in Vascular Surgery. 28: 21-28.

14 Salgueiro S.R., Núñez L.G., Herrera G.D.B., Febles E.S., Ares D.M., Lopez R.M., Acosta JB. 2014. Role of epidermal growth factor and growth hormone-releasing peptide-6 in acceleration of renal tissue repair after kanamycin overdosing in rats. Iranian Journal of Kidney Diseases. 8: 382-388.

15 Shepler B., Nash C., Smith C., Dimarco A., Petty J. \& Szewciw S. 2012. Update on potential drugs for the treatment of diabetic kidney disease. Clinical Therapeutics. 34: 1237-1246.

16 Stojiljković N., Veljković S., Mihailović D., Stoiljković M., Ranković G., Jovanović I. \& Randjelović P. 2009. Pentoxifylline ameliorates glomerular basement membrane ultrastructural changes caused by gentamicin administration in rats. Bosnian Journal of Basic Medical Sciences. 9: 239-244.

17 Stojiljkovic N., Veljkovic S., Mihailovic D., Stoiljkovic M., Radenkovic M., Rankovic G. \& Randjelovic P. 2009. Protective effects of pentoxifylline treatment on gentamicin-induced nephrotoxicity in rats. Renal Failure. 31: 54-61.

18 Turgut K. 2000. Liver disorders and tests. In: Veterinary Clinic Laboratory Diagnosis. Konya: Bahcivanlar Press, pp.202-57.

19 Turgut K. 2000. Erythrocyte disorders and tests. In: Veterinary Clinic Laboratory Diagnosis. Konya: Bahcivanlar Press, pp.17-78 
20 Turgut K. 2000. Endocrine, metabolic and lipid metabolism disorders and tests. In: Veterinary Clinic Laboratory Diagnosis. Konya: Bahcivanlar Press, pp.416-87.

21 Vidal L., Gafter-Gvili A., Borok S., Fraser A., Leibovici L. \& Paul M. 2007. Efficacy and safety of aminoglycoside monotherapy: systematic review and meta-analysis of randomized controlled trials. Journal of Antimicrobial Chemotherapy. 60: 247-257.

22 Wargo K.A. \& Edwards J.D. 2014. Aminoglycoside induced nephrotoxicity. Journal of Pharmacy Practice. 27: 573577.

23 Yazar E. 2010. Rodents. In: Maden M. (Ed). Feeding and Diseases of Exotic Animals. Konya: Erman Press pp.111127.

24 Yazar E., Col R., Uney K., Atalay B., Elmas M. \& Tras B. 2004. Effect of pentoxıfylline on biochemical parameters in endotoxaemic New Zealand white rabbits. Bulletin of the Veterinary Institute in Pulawy. 48: 297-299.

25 Yazar E., Elmas M., Altunok V., Sivrikaya A., Oztekin E. \& Birdane Y.O. 2003. Effects of aminoglycoside antibiotics on renal antioxidants, malondialdehyde levels, and some serum biochemical parameters. Canadian Journal of Veterinary Research. 67: 239-240.

26 Yiannakopoulou E., Nikiteas N., Perrea D. \& Tsigris C. 2012. Pharmacological Modulation of Oxidative Stress Response in Minimally Invasive Surgery. Surgical Laparoscopy Endoscopy \& Percutaneous Techniques. 22: 200-204.

27 Yurt A.O. 2012. Effect of meloxicam on serum vitamin and cytokine levels during endotoxemia. Eurasian Journal of Veterinary Sciences. 28: 47-53. 